\title{
Analysis of Vibration Characteristics of Centrifugal Pump Mechanical Seal under Wear and Damage Degree
}

\author{
Yin Luo $\mathbb{B D}^{1}{ }^{1}$ Wenqi Zhang, ${ }^{1}$ Yakun Fan, ${ }^{1}$ Yuejiang Han, ${ }^{1}$ Weimin Li, \\ and Emmanuel Acheaw ${ }^{1}$ \\ ${ }^{1}$ Research Center of Fluid Machinery Engineering and Technology, Jiangsu University, Zhenjiang 212013, China \\ ${ }^{2}$ Shenzhen College of Advanced Technology, University of Chinese Academy of Sciences, Shenzhen 518055, China \\ Correspondence should be addressed to Yin Luo; luoyin@ujs.edu.cn
}

Received 7 November 2020; Revised 31 December 2020; Accepted 24 March 2021; Published 8 April 2021

Academic Editor: Mohamed A. A. Abdelkareem

Copyright (c) 2021 Yin Luo et al. This is an open access article distributed under the Creative Commons Attribution License, which permits unrestricted use, distribution, and reproduction in any medium, provided the original work is properly cited.

\begin{abstract}
Mechanical seal is a kind of shaft sealing equipment. Face wear is one of the main causes of mechanical seal failure. Mechanical seal condition is also related to the reduction of energy consumption and carbon emission. Therefore, we need to detect the centrifugal pump seal condition. At present, vibration signal is a common method for fault monitoring and diagnosis of centrifugal pump. In this paper, the vibration signal under the condition of damaged centrifugal pump seal is measured by studying the characteristics of vibration signal after the end face damage of centrifugal pump. Statistical indicators such as RMS and kurtosis were taken to analyze the average energy and shock wave energy of vibration signal. The time-frequency characteristics of vibration signal are analyzed by frequency spectrum. The results show that there are a large extent variation of vibration amplitude in the direction of base and axis and a weak variation of vibration amplitude in the direction of radial and vertical. With the increasing of flow rate, the RMS of vibration signal falls at first, then keeps steady, and mounts at last when the flow rate is over the design flow rate. It can be shown from the time-frequency spectrum that there is a shock wave and pause signals caused by the shock wave, which are reflected by the higher frequency band components of the vibration signal that can provide a reference to the diagnosis of the occurrence of damaged mechanical seal. From the analysis, the energy of vibration signal is related to the running condition, we can find that the occurrence of mechanical seal wear makes the centrifugal pump to produce high-frequency vibration signal, and the axial vibration is the strongest and the instability in the fluid makes the vibration signal produce high amplitude characteristics. Analyzing the vibration signal characteristics of centrifugal pumps with damaged mechanical seal is of great significance to find the mechanical seal failure of the centrifugal pumps and adjust the operating parameters.
\end{abstract}

\section{Introduction}

Centrifugal pump is an important energy conversion and fluid transportation device [1]. Mechanical seal is mainly composed of moving ring, static ring, compress element, and sealing element. Failure of mechanical seal is one of the common faults of centrifugal pump. Detection of mechanical seal failure can realize early warning of faults and make corresponding adjustments to the operation mode of the pump and its system to reduce the frequency of mechanical seal damage and other related faults, thus ensuring the longterm stable operation of the equipment, guarantee the normal use of centrifugal pump, reducing the consumption of spare parts and labor costs, and achieve certain economic benefits.

Rotating machinery is of vital importance in the field of engineering [2]. Fault diagnosis of rotating machinery plays a significant role in the industrial production and engineering field [3]. The vibration characteristics of centrifugal pump during operation are related to the type, location, and cause of the fault. It is a common method to monitor and diagnose the fault of centrifugal pump by using vibration signal. The bearing consists of an inner ring, an outer ring, a support frame, and a rotating part. Each component may have fault and produce high-frequency vibration, which could be analyzed by vibration signal [4]. It can predict and 
diagnose the faults on the inner ring, outer ring, and rotor components of the rolling axle load by determining [5]. Research members domestic and abroad have adopted a series of methods to detect the mechanical seal damage. Li et al. [6] proposed a new approach based on genetic particle filter with autoregression can effectively detect the contact state of the seal end faces and has higher accuracy rates than some other existing methods. Sun et al., based on the analysis of the stationary ring's output response results, obtained the parameter selection scheme of noncontact mechanical seals at high and low frequencies [7].

Unsworth et al. [8] analyzed the frequency spectrum of motor current and voltage signal through Fourier transform and realized the fault diagnosis of centrifugal pump seal. Daraz et al. [9] used modulation signal spectrum to identify the seal failure as it has the unique capability to demodulate the signal along with noise reduction. Anderson et al. [10] presented a monitoring system for liquid-lubricated seals that avoids the problems with $\mathrm{AE}$ and other monitoring systems. Cimbura [11] presented invention provides a shaft seal that can operate remotely and monitor itself for failure. Vibration analysis is necessary to detect and diagnose faults of the pumping stations and to avoid any failure or malfunction [12]. Birajdar et al. [13] studied the fault diagnosis of impeller damage by means of sound pressure level and vibration spectrum analysis. Similar to vibration signal, analysis of electrical signal is also a noninvasive method for equipment fault diagnosis. Hernandez and Parlos [14] used power spectral density to analyze the frequency domain of motor current and power signal and analyze the current characteristics of motor stator under different mechanical faults of centrifugal pump and realized the fault diagnosis of centrifugal pump cavitation and impeller wear. Zhu et al. [15] studied that the sealing performance is enhanced by increasing the spacing of adjacent sealing sheets. Sakthivel et al. [16] presented the use of decision tree algorithm for fault diagnosis through statistical feature extracted from vibration signals of good and faulty conditions. Awad et al. aimed at the problem that the annular seal of ball valve in hydropower plant sometimes does not work and proposed a model which can explain the excitation mechanism and estimate the hydrodynamic behavior [17]. Fribourg et al. found that cooling pumps in high-power diesel engines will experience high-level cooling vibration, which can lead to mechanical seal problems in these pumps. Test equipment is proposed to investigate this problem and obtain test results, which can be widely used in mechanical seal design [18]. Yan et al. revealed the evolutionary process of vortex shedding and the flow law around airfoils by superior performance [19]. Multiphase flow calculation and high-speed photography technology have been used to study the entrainment of gas-solid flow under different inlet flow rates [20]. Based on the research method combining numerical calculation and experiment, Yang et al. studied a typical mixed-flow ESP and obtains the pressure pulsation law inside the first, second, and third stages [21].

Until now, there are many research studies on the rigid flexible coupling of friction pair and bellows, but few research studies on the wear of moving ring and static ring.
The main damage forms of mechanical seal are the wear of seal face and rupture of dynamic and static rings. However, for the mechanical seal system, the oscillatory properties of related aspects are studied, mainly taking the shafting vibration as the main experimental direction, or only for the vibration properties of a single component, but in the overall mechanical seal system, the research work on vibration problems is not enough.

This paper mainly studies the vibration characteristics of the centrifugal pump under the normal and damaged conditions of the dynamic ring and the static ring of the mechanical seal. According to experiment, the change in vibration character causes damage in mechanical seal of centrifugal pump, which serves as a reference for understanding the vibration signal of the pump and predicting the degree of damage on the mechanical seal.

\section{Apparatus and Experiments}

As shown in Figure 1, a closed-type experiment rig consists of tank body, inlet and outlet pipes, electric valve, vacuum pump, motor, electromagnetic flowmeter, and pressure sensor. For the collection of experimental data, flow rate, inlet pressure, outlet pressure, vibration acceleration signal, vibratory-accelerated sensor are used to get the collection of vibration information of pump body. The sensor has a measuring range of 5-60 Hz and sensitivity of $1017 \mathrm{mV} \cdot\left(\mathrm{m}^{2} / \mathrm{s}^{2}\right)$. Acquisition card USB6364 has been used to transfer data to the computer. The processing of test data and the operation of the test system are controlled by a computer.

In order to study the vibration rule of the centrifugal pump with damaged mechanical seal, Ns $=88$, IS-50-160-00 standard centrifugal pump was machined, and its basic design parameters are as follows: flow rate $Q$ is $50 \mathrm{~m}^{3} / h$, lift $\mathrm{H}$ is $34 \mathrm{~m}$, rated speed $\mathrm{N}$ is $2900 \mathrm{rpm}$, and efficiency is $72.8 \%$. This paper mainly studies the characteristics of different mechanical seals, selecting two mechanical seals of the same material and model, the left two are the static ring and the moving ring of the normal mechanical seal, the right two are the static ring and the moving ring of the damaged mechanical seal, and the static ring of the damaged one is badly damaged, and the moving ring has broken. We install two seals in the centrifugal pump for performance test experiment.

Mechanical seal wear monitoring is mainly based on vibration signals, centrifugal pump head, and efficiency. Vibration and noise are used to ensure the operation status to monitor accuracy and betimes. The centrifugal pump seal wear test is carried out by measuring the pump's flow, inlet and outlet pressure, vibration signal, and other physical quantities under different working conditions. These measurable quantities are used to analyze and compare the variation of pump performance curve after the test and the characteristics of vibration signal after machine seal wear in time domain and frequency spectrum under different flow rates, in order to establish the relationship between mechanical seal wear and these characteristics. The experiment is conducted on the test bench as shown in Figure 1(a). Figure 1(b) shows the overall test, and Figure 1(c) shows the 


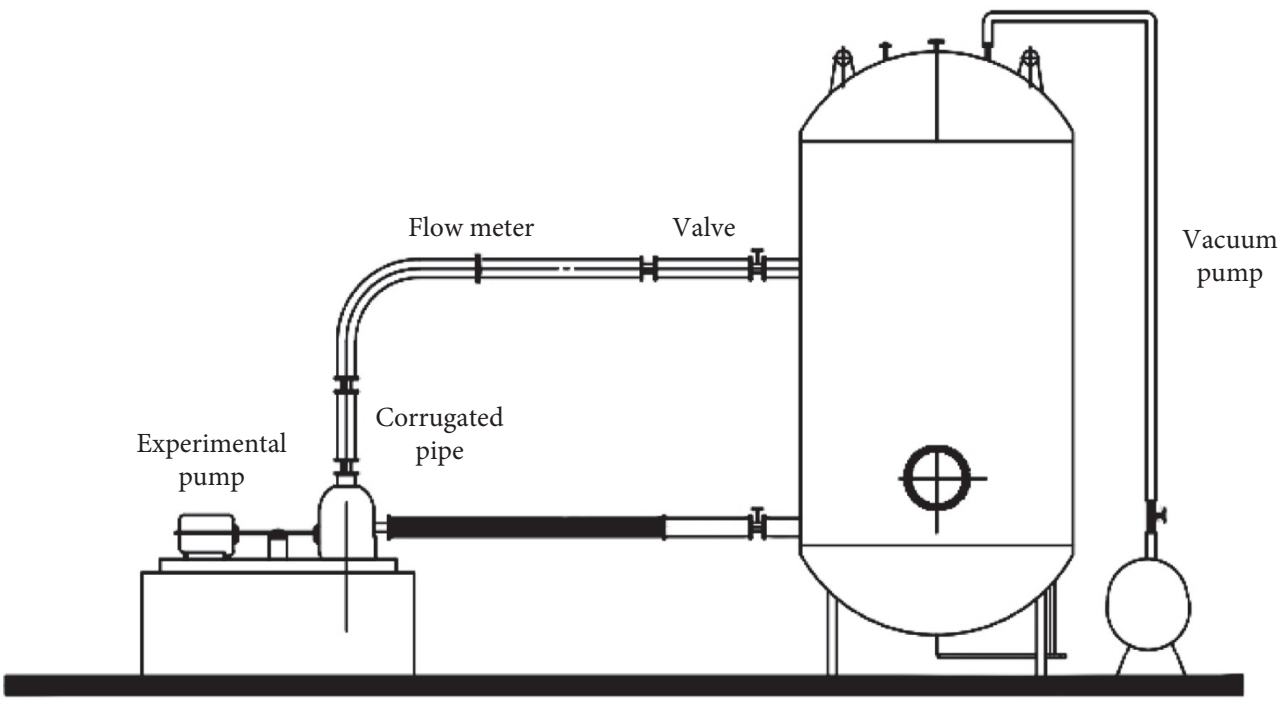

(a)

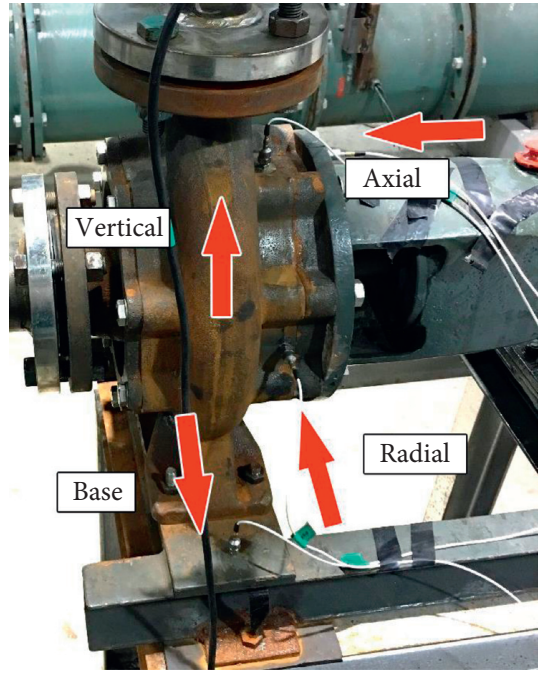

(b)

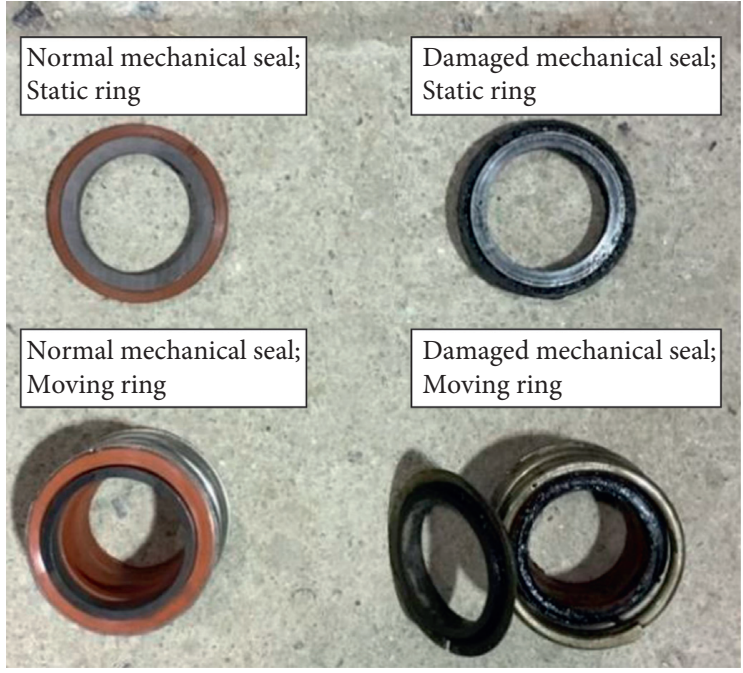

(c)

Figure 1: Test device. (a) Test circuit. (b) Test-bed. (c) Mechanical seal.

mechanical seal selected for the test. The result from the analysis shows that the pump system leaks due to the damage of the machine seal, and the internal flow of the pump is unstable, which leads to the lift and efficiency of the centrifugal pump head decreases, but the shaft power has no obvious change.

\section{Experiment Results and Analysis}

3.1. Pump Performance Test under Different Seal Failure. According to the definition of pump head and efficiency, the test obtained the data of wheel head and hydraulic efficiency of centrifugal pump with normal seal end face and damaged seal end face under each flow rate, and the performance curve is drawn as shown in Figure 2. By comparing the performance curve of the damaged mechanical seal with that of the normal mechanical seal, it could be seen that the wear of the mechanical seal leads to the reduction of head and efficiency. The main reason for this phenomenon is that the head and efficiency of the pump are reduced due to the increased flow instability of the pump after sealing wear.

\subsection{Analysis and Calculation of Experiment Uncertainty.} Due to the imperfect of the experimental method and equipment, the influence of the surrounding, instrument error, visual inspection, measurement procedure, and measurement error of data is inevitable. Therefore, measurement uncertainty is used to characterize the magnitude of error. Uncertainty is divided into two categories: random uncertainty and systematic uncertainty. Systematic errors are mainly caused by the limitations of measurement tools or methods. Accordingly, the systematic errors can be reduced by improving the precision of the measuring instrument and the measurement method. The random errors are reduced mainly by increasing the 


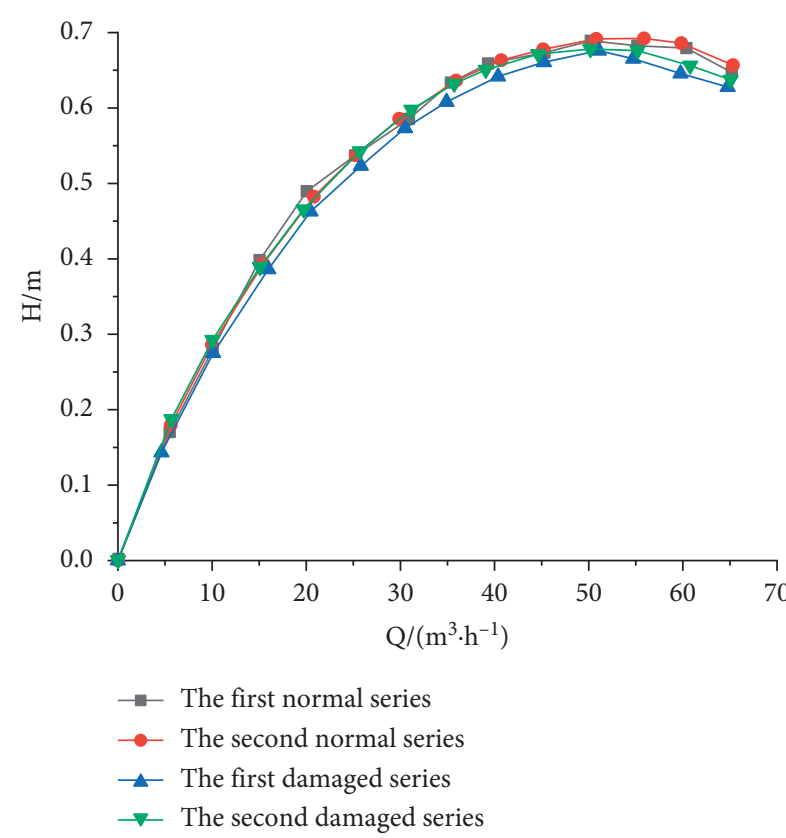

(a)

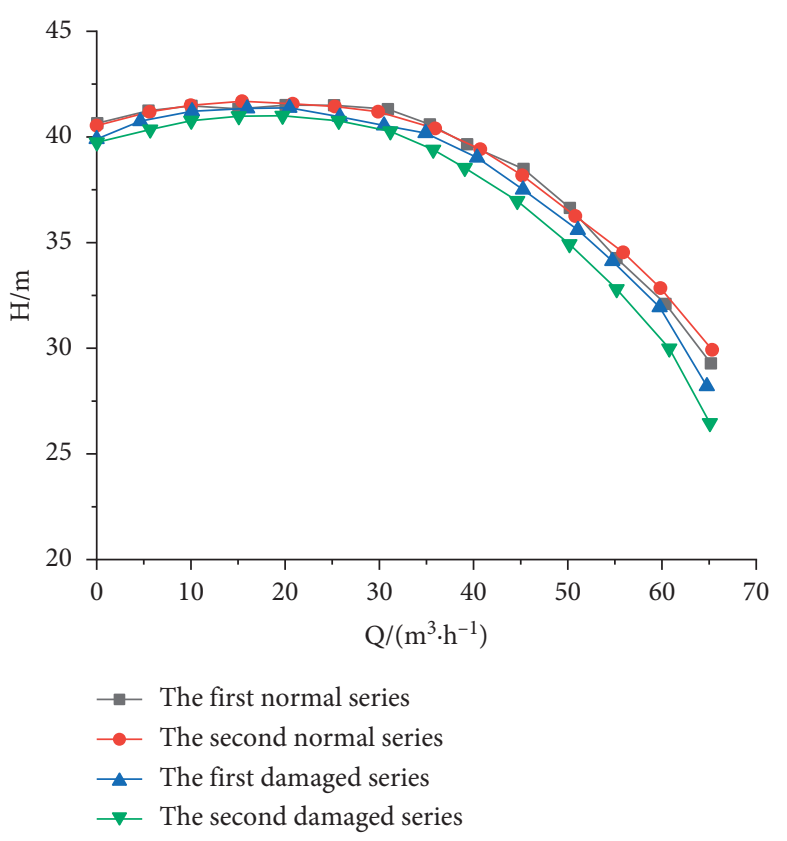

(b)

Figure 2: Test device. (a) Head diagram. (b) Efficiency diagram.

number of measurements. The value of uncertainty can be used to judge the accuracy and reliability of measurement results.

Random uncertainty can be expressed by relative uncertainty $\mathrm{E}_{\mathrm{R}}[22]$ :

$$
E_{R}= \pm \frac{t_{n-1} S_{x}}{\bar{x} \sqrt{n}} \times 100 \% .
$$

In the formula, $t_{n-1}$ is confidence coefficient and the confidence coefficient of $95 \%$ confidence probability is generally adopted.

3.2.1. The Synthesis of Random Uncertainty. At the design condition, the random uncertainty of flow is

$$
E_{Q, R}= \pm \frac{t_{n-1} S x}{\bar{Q} \sqrt{n}}= \pm \frac{1.96 \times 0.6303}{49.70 \times \sqrt{10000}}= \pm 0.025 \% \text {. }
$$

The random uncertainty of head:

$$
E_{H, R}= \pm \frac{t_{n-1} S x}{\bar{H} \sqrt{n}}= \pm \frac{1.96 \times 0.06235}{32.41 \times \sqrt{10000}}= \pm 0.037 \% .
$$

The random uncertainty of torque:

$E_{T, R}= \pm \frac{t_{n-1} S x}{\bar{T} \sqrt{n}}= \pm \frac{1.96 \times 0.055}{22.36 \times \sqrt{10000}}= \pm 0.0048 \%$.

The random uncertainty of rotate speed:

$E_{T, R}= \pm \frac{t_{n-1} s_{x}}{\bar{T} \sqrt{n}}= \pm \frac{1.95 \times 2.56}{2971.49 \times \sqrt{10000}}= \pm 0.0016 \%$.

The random uncertainty of efficiency:

$$
E_{\eta, R}= \pm \sqrt{E_{Q, R}^{2}+E_{H, R}^{2}+E_{n, R}^{2}+E_{T, R}^{2}}= \pm 0.049 \% \text {. }
$$

3.2.2. The Synthesis of Systematic Uncertainty. The systematic uncertainty mainly depends on the uncertainty of measuring instrument.

The systematic uncertainty of the turbine flowmeter used in this experiment is

$$
E_{\mathrm{Q}, S}= \pm 0.2 \% .
$$

Torque is directly reading amount by the instrument, which depends on the test instrument used for the test:

$$
E_{Q, S}= \pm 0.1 \% \text {. }
$$

Uncertainty of rotate speed: in the test, using LabVIEW to measure the rotation speed, the reference clock accuracy of LabVIEW is $50 \mathrm{ppm}\left(5 \times 10^{-5}\right)$; after calculation, the error caused by this method is $1 \times 10^{-7}$ :

$$
E_{\eta, S}= \pm \sqrt{\left(5 \times 10^{-5}\right)^{2}+\left(1 \times 10^{-7}\right)^{2}}= \pm 0.055 \% .
$$

Uncertainty of head: the calculation of head is the accuracy of inlet and outlet pressure sensor, which is 0.25 level:

$$
E_{P, S 1}=E_{P, S 2}= \pm 0.025 \% \text {. }
$$

Systematic uncertainty of the head measured by the two pressure gauges:

$$
E_{p, s}= \pm \sqrt{E_{p, s 1}^{2}+E_{p, s 2}^{2}}= \pm 0.35 \% .
$$

The uncertainty of efficiency: 


$$
E_{\eta, R}= \pm \sqrt{E_{Q, R}^{2}+E_{H, R}^{2}+E_{n, R}^{2}+E_{T, R}^{2}}= \pm 0.4154 \% \text {. }
$$

3.2.3. The Synthesis of Overall Uncertainty. The overall uncertainty of the flow:

$$
E_{Q}= \pm \sqrt{E_{Q, S}^{2}+E_{Q, R}^{2}}= \pm \sqrt{0.2 \%^{2}+0.025 \%^{2}}= \pm 0.2 \% .
$$

The overall uncertainty of the head:

$$
E_{H}= \pm \sqrt{E_{H, S}^{2}+E_{H, R}^{2}}= \pm \sqrt{0.035 \%^{2}+0.037 \%^{2}}= \pm 0.051 \% .
$$

The overall uncertainty of the rotate speed:

$E_{n}= \pm \sqrt{E_{n, S}^{2}+E_{n, R}^{2}}= \pm \sqrt{0.005 \%^{2}+0.0016 \%^{2}}= \pm 0.0052 \%$.

The overall uncertainty of the efficiency:

$E_{\eta}= \pm \sqrt{E_{\eta, S}^{2}+E_{\eta, R}^{2}}= \pm \sqrt{0.049 \%^{2}+0.4154 \%^{2}}= \pm 0.4182 \%$.

Through the calculation of the above uncertainty, it can be found that the uncertainty of the test data is small, which further indicates that the test results are stable and reliable.

\section{Time-Domain Analysis of Experiment Data}

4.1. Analysis of Experiment Data in Time-Domain Results. The time-domain processing is mainly about waveform processing of vibration signals, and the filtering is the main way to process signals. Firstly, MATLAB is used for linear average of data to remove the interference of random signals. Then, four digital filters with low pass, high pass, band pass, and band stop functions are used. Figure 3 shows the timedomain characteristics of the axial vibration signal. Comparing the vibration signals before and after the mechanical seal damaged, it was found that the axial vibration signals were significantly enhanced after the mechanical seal has been damaged, and the radial, base, and longitudinal trends were small. As Figure 3 shows, the axial vibration amplitude of the centrifugal pump is large after the mechanical seal is damaged, and all of them are irregular vibration. The maximum extreme value is hundreds of times of the normal machine extreme value.

4.2. Statistical Analysis of Experiment Data. RMS analysis is commonly used for analyzing data in signal processing, mainly analyzing the average effective energy of the signal.

RMS:

$$
T_{\max }=\sqrt{\frac{1}{N} \sum_{K=1}^{N} X_{K}^{2}} .
$$

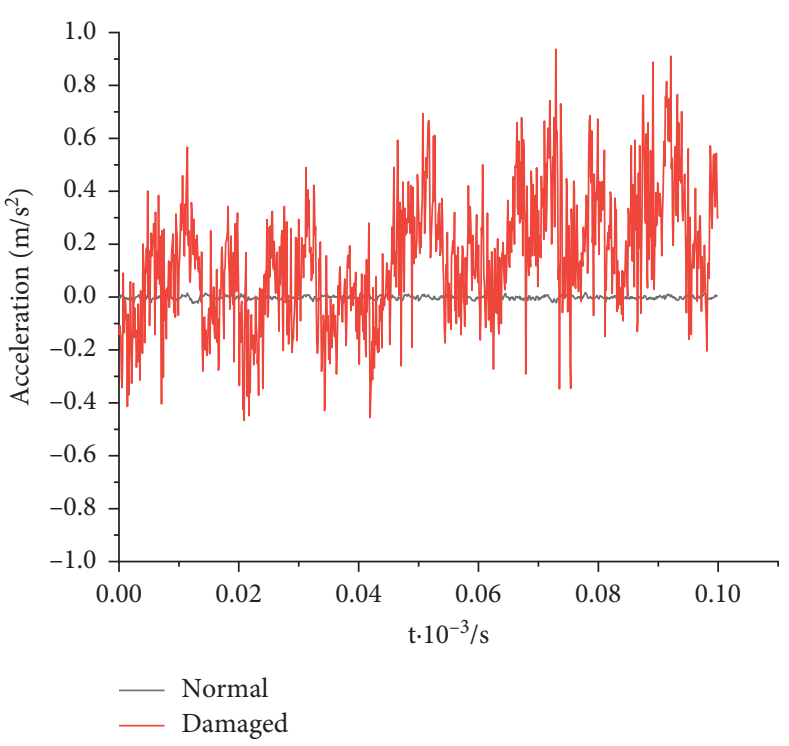

FIgURe 3: Axial vibration time-domain diagram of vibration signal.

In this formula, $X_{K}$ is measurement value of vibration signal, $K=1,2,3, \ldots \ldots, N$ [23].

Figure 4 shows the vibration energy curve of different damage seal and healthy seal in the whole working condition range. The ordinate in the figure represents the root mean square of the vibration acceleration and the abscissa represents the full flow for each experiment.

According to the four curves, the overall trend is with the increase of flow rate, and the vibration energy of the damaged mechanical seal decreases first and then increases. Figure 4 shows the axial direction vibration intensity is the most, the vibration intensity of base, radial, and longitudinal decreases successively, and the longitudinal vibration intensity is the weakest. The axial variation is mainly affected by the impact of the flowing water and leakage of the pump system due to the damage of the mechanical seal, resulting in unstable flow inside the pump and uneven distribution of pressure causes more intense vibration.

With the increase of the flow rate, the average energy of the vibration signals before and after mechanical seal wear shows the trend of decreasing first and increasing later, then staying flat, and then rising again. The main reason is that when the centrifugal pump is running at a low flow rate, there will be secondary back in the pump. The repeated collision between the secondary back liquid and the outlet liquid makes the centrifugal pump impacted by the liquid and produces a strong vibration. When the flow rate of the centrifugal pump increases, the flow in the pump tends to be stable, and then the vibration intensity of the centrifugal pump decreases. The analysis shows that for small flow rates, the pump flow is very unstable with significant backflow and intense local abrasion, and as the particle concentration increases, the flow resistance increases $[24,25]$. At low flow rate, centrifugal pump vibration is mainly caused by fluid flow instability induced vibration. With the increase of flow, the flow in the pump tends to be stable, and the balance 


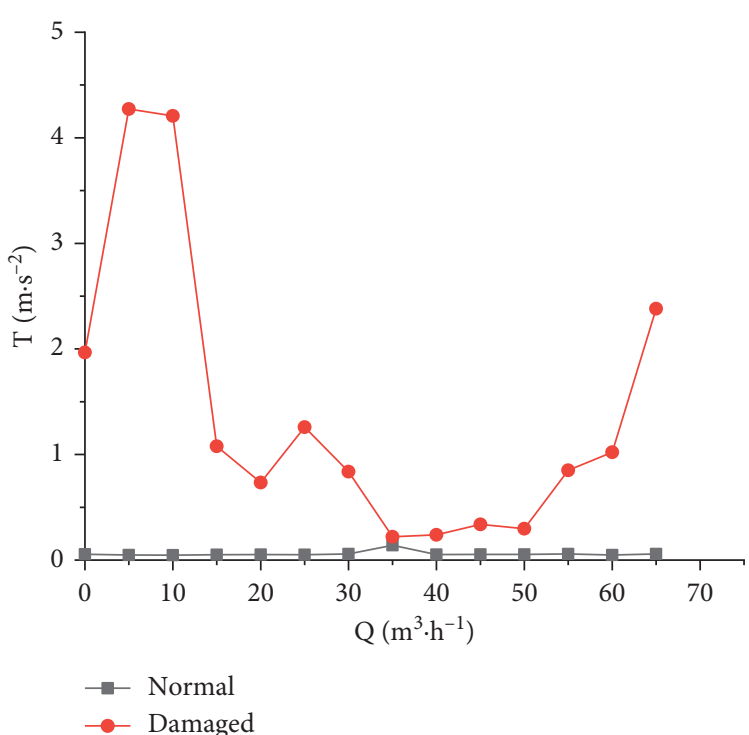

(a)

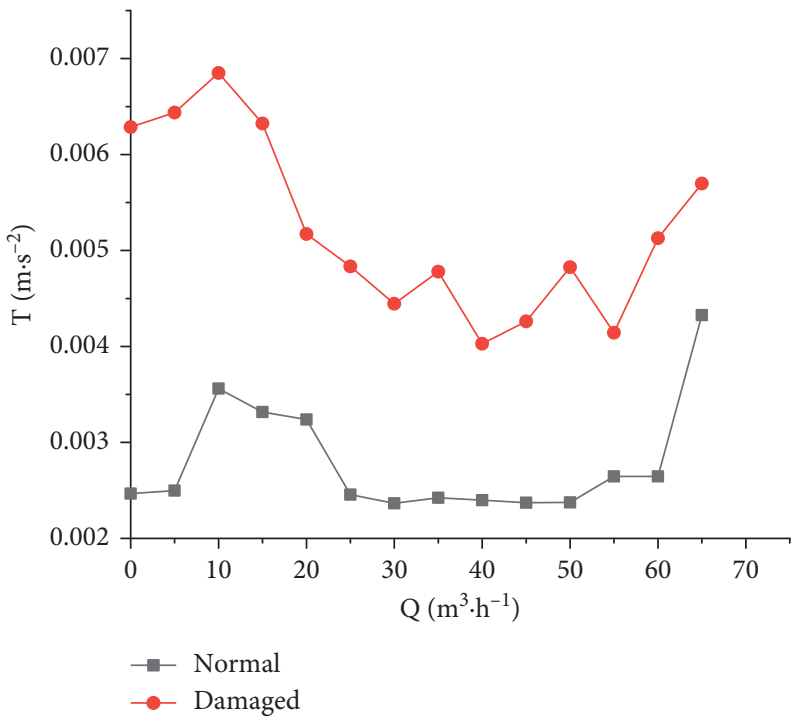

(c)

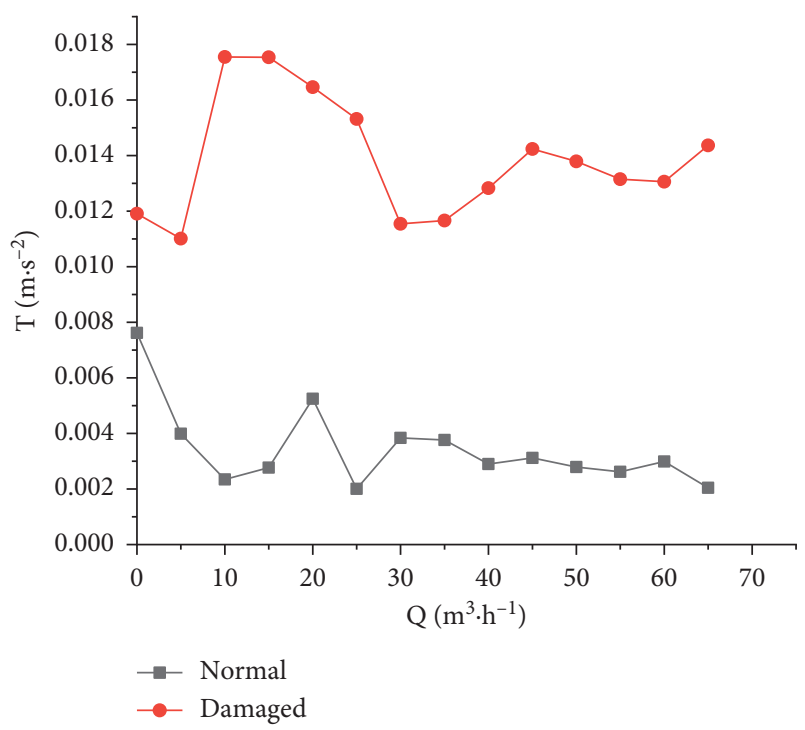

(b)

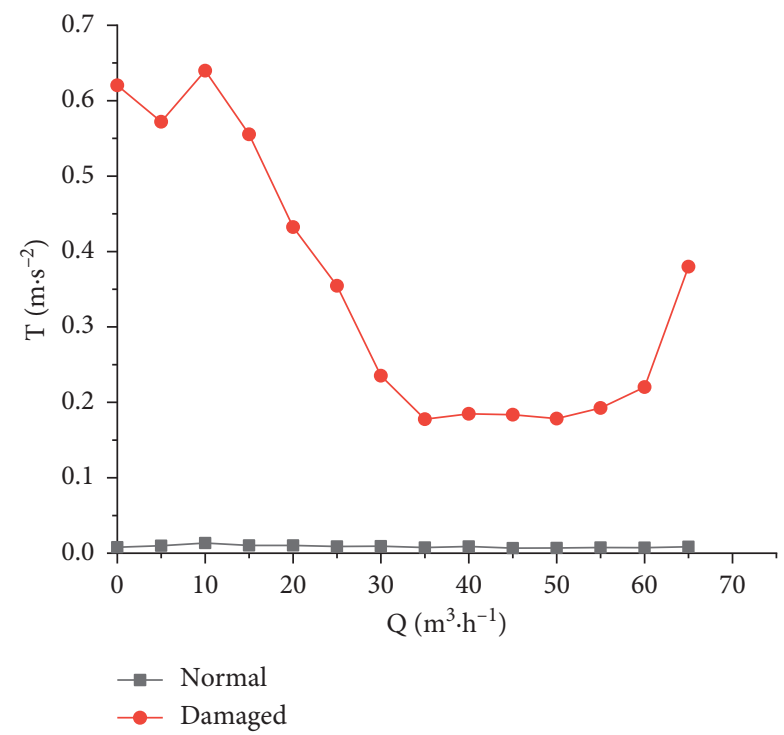

(d)

FIgURE 4: The vibration intensity varies with the flow curve. (a) Base RMS of vibration signal. (b) Radial direction RMS of vibration signal. (c) Axial direction RMS of vibration signal. (d) Vertical direction RMS of vibration signal.

between the fluid-induced vibration decline and the mechanical vibration increase caused by the increase of flow makes the vibration of the whole centrifugal pump change in a small range.

\section{Spectrum Analysis of Test Data}

Using the power spectrum to analyze the vibration signal can determine the optimum working parameters of the pump and find the faults and positions in the pump. Power spectrum is the average value of energy flow in unit time and frequency unit interval in the whole-time process. MATLAB was used to estimate the autocorrelation of the filtered vibration signal, and then we used fast Fourier transform; finally, the power spectrum of the vibration signal was obtained by using the power spectrum function.

The vibration power spectrum is shown in Figure 5. Mechanical seal wear has a great influence on the vibration spectrum analysis of centrifugal pump. The power spectrum amplitudes of normal seal and damaged seal are different in magnitude. According to the test results, the vibration signal energy of the damaged mechanical seal reached the maximum at $300-500 \mathrm{~Hz}$. The power spectrum of the pump was usually in a stable state at $1000-5000 \mathrm{~Hz}$. It can be seen from Figure 5(a) that the vibration signals in the base direction are relatively scattered. The vibration in the direction of the base was caused by interaction of many factors, such as blade frequency and unbalanced impeller, rotor unbalance, and others, which causes relatively dispersed low frequency 

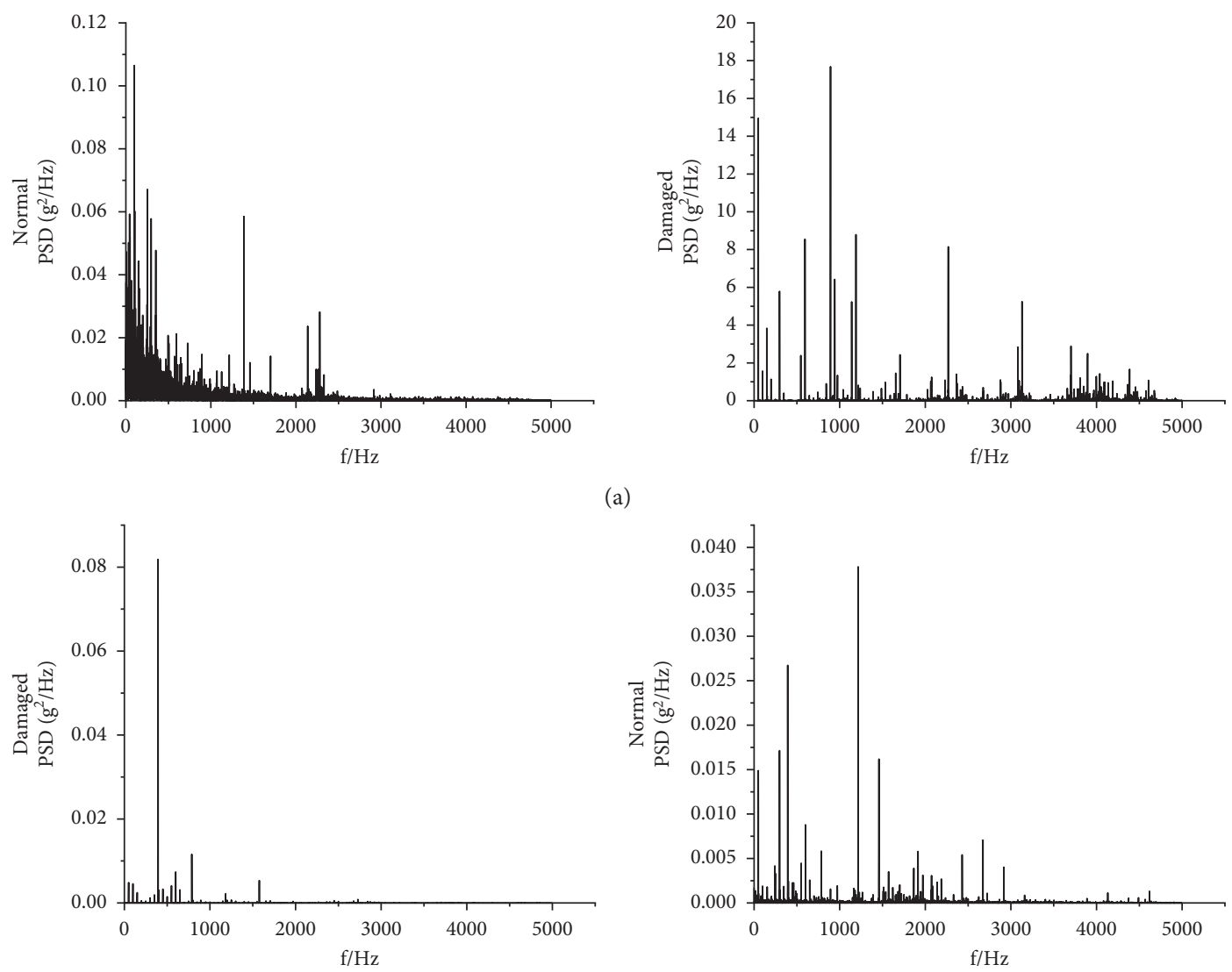

(a)

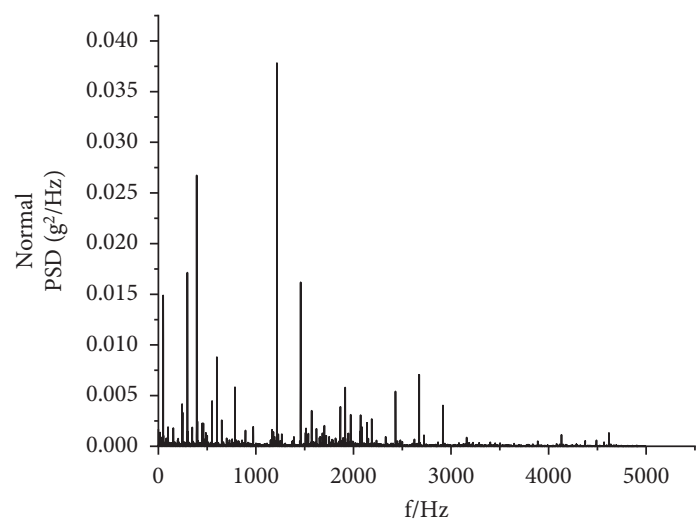

(b)
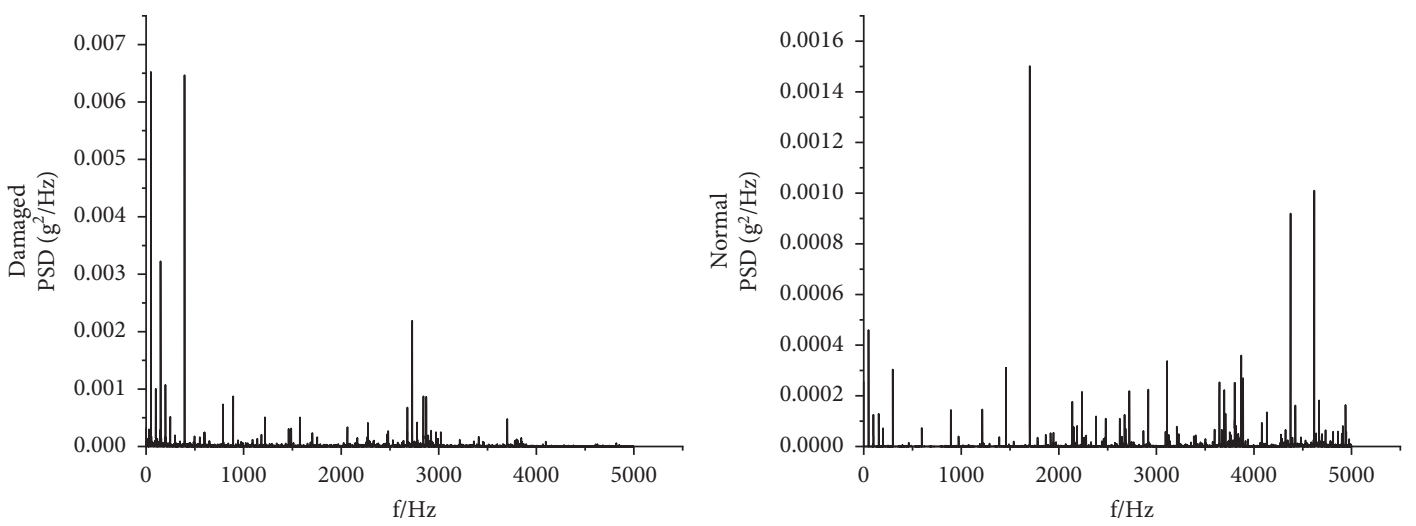

(c)
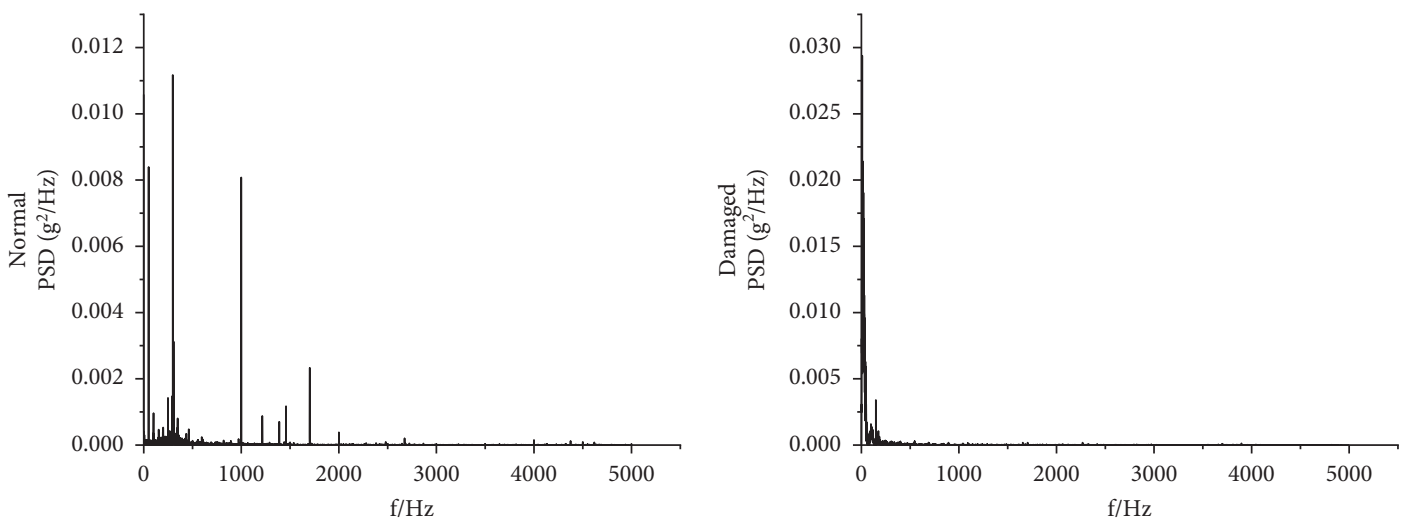

(d)

FIGURE 5: Vibration power spectrum. (a) Base direction power spectrum of vibration signal. (b) Radial direction power spectrum of vibration signal. (c) Vertical direction power spectrum of vibration signal. (d) Axial direction power spectrum of vibration signal. 
vibration. The vibration signals from Figure 5(d) are more concentrated than those from Figure 5(a) and are concentrated in the blade frequency accessories. Axial vibration was mainly caused by the reaction of fluid on the blade when the blade worked on the fluid. Other vibration signals had little influence on axial vibration. Therefore, axial vibration signals were concentrated near the blade frequency. The reasons for the occurrence of high-frequency vibration signals after mechanical seal has been damaged lie in the flow instability of the fluid enhanced in case of leakage due to mechanical seal wear, which leads to the asymmetric field on the impeller and pump body, thus inducing the high-frequency vibration. Under the condition of mechanical seal, strong wave and microjet produced high-frequency vibration. At the same time, the sensor may obtain massive pulse signals. Therefore, it can be observed that the vibration intensity of high-frequency signals in the same frequency band along the direction of time was strong or weak. Mechanical seal wear also has induced high-frequency vibration of other parts, which has been transmitted to the base and reflected in the time-frequency diagram, resulting in appearance of multiple discrete high-frequency components.

Comparing the longitudinal vibration signals before and after the mechanical seal failure, it can be seen that the mechanical seal failure causes high-frequency vibration signals in the longitudinal direction, which are concentrated at $1500-2500 \mathrm{HZ}$.

According to the time-frequency analysis, when the mechanical seal is not damaged, the vibration signals are mainly concentrated at low frequency, mainly below $1000 \mathrm{HZ}$. After the mechanical seal is damaged, high-frequency vibration signals are generated in both the base direction and the longitudinal direction, which are affected by many factors, while the longitudinal direction is mainly concentrated at 1500-2500 HZ. The high-frequency vibration signals can provide reference for fault diagnosis of mechanical seal.

\section{Conclusion}

In this paper, the centrifugal pump is taken as the research object, the test data are collected and processed by LabVIEW, and the vibration acceleration signals collected in the test are analyzed in time domain and frequency spectrum, and the following conclusions are drawn:

(1) The measurement of vibration signal is related to the installation position of the sensor. Comparing the vibration signals at the base, vertical, axial, and base directions, it can be seen that axial vibration is stronger and it is greatly affected by mechanical seal wear. The energy of vibration signal is related to the running condition; when the flow rate is lower than $0.3 \mathrm{Q}$, the instable fluid flow will cause stronger vibration. When the flow rate exceeds the design condition, the mechanical vibration is strengthened, resulting in the rapid increase of vibration energy in the direction of the base.
(2) In time-domain analysis, the axial vibration is the strongest and the instability in the fluid makes the vibration signal produce high amplitude characteristics.

(3) The occurrence of mechanical seal wear makes the centrifugal pump to produce high-frequency vibration signal. There is almost no vibration signal higher than $1000 \mathrm{~Hz}$ in centrifugal pump before the mechanical seal wear, but massive high-frequency vibration signals are generated after mechanical seal wear. High-frequency vibration signals higher than 1 $000 \mathrm{~Hz}$ can provide certain reference value for mechanical seal diagnosis of centrifugal pump.

\section{Data Availability}

The data used to support the findings of this study are available from the corresponding author upon request.

\section{Conflicts of Interest}

The authors declare no conflicts of interest.

\section{Authors' Contributions}

Y.L carried out conceptualization. Y.L. and W.Z. were responsible for methodology and software, formal analysis, investigation, data curation, and writing: original draft preparation; formal analysis was conducted by Y.L and W.Z.; project administration was done by Y.L. All the authors have read and agreed to the published version of the manuscript.

\section{Acknowledgments}

The authors would like to acknowledge the funding sponsored by the National Natural Science Foundation of China (grant no. 51979127), the Nature Science Foundation of Jiangsu Provience (grant no. BK20171403), and National Key Research and Development Program of China (grant no. 2016YFD0700602).

\section{References}

[1] H. Sun, S. Yuan, Y. Luo et al., "Unsteady flow analysis of centrifugal pumps influenced by flow, motor and electricity," Journal of Drainage and Irrigation Machinery Engineering, vol. 34, no. 2, pp. 122-127, 2016.

[2] S. Tang, S. Yuan, and Y. Zhu, "Convolutional neural network in intelligent fault diagnosis toward rotatory machinery," IEEE Access, vol. 8, pp. 86510-86519, 2020.

[3] S. Tang, S. Yuan, and Y. Zhu, "Deep learning-based intelligent fault diagnosis methods toward rotating machinery," IEEE Access, vol. 8, pp. 9335-9346, 2019.

[4] J. S. Rao, Vibratory Condition Monitoring of machines, CRC Press, Boca Raton, FL, USA, 2000.

[5] M. Amarnath, R. Shrinidhi, A. Ramachandra et al., "Prediction of defects in antifriction bearings using vibration signal analysis," Journal of the Institution of Engineers (India), Part MC, Mechanical Engineering Division, vol. 85, p. 88, 2004. 
[6] X. Li, P. Fu, K. Chen et al., "The contact state monitoring for seal end faces based on acoustic emission detection," Shock and Vibration, vol. 2016, Article ID 8726781, 8 pages, 2016.

[7] D. F. Sun, J. J. Sun, C. B. Ma et al., "Frequency-domain-based nonlinear response analysis of stationary ring displacement of noncontact mechanical seal," Shock and Vibration, vol. 2019, Article ID 7082538, , 2019.

[8] P. J. Unsworth, F. M. Discenzo, and V. S. Babu, "Detection of pump cavitation/blockage and seal failure via current signature analysis," U.S. Patent 6,757,665, 2004.

[9] A. Daraz, S. Alabied, D. Zhen et al., "Detection and diagnosis of mechanical seal faults in centrifugal pumps based on acoustic measurement," in Advances in Asset Management and Condition Monitoring,Smart Innovation, Systems and Technologies, pp. 963-975, Springer, Cham, Switzerland, 2020.

[10] W. B. Anderson, J. Jarzynski, and R. F. Salant, "A condition monitor for liquid lubricated mechanical seals," Tribology Transactions, vol. 44, no. 3, pp. 479-483, 2001.

[11] J. A. Cimbura Sr, "Selfmonitoring oil pump seal," U.S. Patent 5,755,372, 1998.

[12] S. M. Abdel-Rahman and S. A. El-Shaikh, "Diagnosis vibration problems of pumping stations: case studies," in Proceedings of the 13th IWTC (International Water Technology Conference), Hurghada, Egypt, March 2009.

[13] R. Birajdar, R. Patil, and K. Khanzode, "Vibration and noise in centrifugal pumps-sources and diagnosis methods," in Proceedings of the $3 r d$ International Conference on Integrity, Reliability and Failure, Porto, Portugal, pp. 20-24, July 2009.

[14] P. P. Harihara and A. G. Parlos, "Sensorless detection of impeller cracks in motor driven centrifugal pumps," in ASME International Mechanical Engineering Congress and Exposition, pp. 17-23, ASME, New York, NY, USA, Article ID 48661, 2008.

[15] H. Zhu, D. Li, H. Pu et al., "Experimental and numerical investigations on the local direct leakage process of rotary regenerative air preheater," Applied Sciences, vol. 10, no. 4, p. $1523,2020$.

[16] N. R. Sakthivel, V. Sugumaran, and S. Babudevasenapati, "Vibration based fault diagnosis of monoblock centrifugal pump using decision tree," Expert Systems with Applications, vol. 37, no. 6, pp. 4040-4049, 2010.

[17] H. Awad and J. Parrondo, "Hydrodynamic self-excited vibrations in leaking spherical valves with annular seal," Alexandria Engineering Journal, vol. 59, no. 3, pp. 1515-1524, 2020.

[18] D. Fribourg, A. Audrain, and L. Cougnon, "The performance of mechanical seals used in a high vibration environment," Sealing Technology, vol. 2010, no. 2, pp. 7-11, 2010.

[19] H. Yan, X. Su, H. Zhang et al., "Design approach and hydrodynamic characteristics of a novel bionic airfoil," Ocean Engineering, vol. 216, Article ID 108076, 2020.

[20] L. Zhou, C. Han, L. Bai, W. Li, M. A. El-Emam, and W. Shi, "CFD-DEM bidirectional coupling simulation and experimental investigation of particle ejections and energy conversion in a spouted bed," Energy, vol. 211, Article ID 118672, 2020.

[21] Y. Yang, L. Zhou, W. Shi, Z. He, Y. Han, and Y. Xiao, "Interstage difference of pressure pulsation in a three-stage electrical submersible pump," Journal of Petroleum Science and Engineering, vol. 196, Article ID 107653, 2021.

[22] M. Kušnerová, J. Valíček, M. Harničárová et al., “The combined relative uncertainty of measurement results by prototype semi-automated calorimetric chamber," Measurement Science Review, vol. 19, no. 2, pp. 53-60, 2019.
[23] L. Ban, W. Du, and C. Qi, “A modified roughness index based on the root mean square of the first derivative and its relationship with peak shear strength of rock joints," Engineering Geology, vol. 279, Article ID 105898, 2020.

[24] G. Peng, X. Huang, L. Zhou, G. Zhou, and H. Zhou, "Solidliquid two-phase flow and wear analysis in a large-scale centrifugal slurry pump," Engineering Failure Analysis, vol. 114, Article ID 104602, 2020.

[25] L. Bai, L. Zhou, X. Jiang, Q. Pang, and D. Ye, "Vibration in a multistage centrifugal pump under varied conditions," Shock and Vibration, vol. 2019, Article ID 2057031, 9 pages, 2019. 